\title{
Effects of Psoralen as an Anti-tumor Agent in Human Breast Cancer MCF-7/ADR Cells
}

\author{
Xiaohong Wang, ${ }^{\text {K Kai Cheng, }}$, Yong Han, Guoqiang Zhang, Jianli Dong, Yuzhen Cui, and \\ Zhenlin Yang* \\ Department of Thyroid and Breast Surgery, The Affiliated Hospital of Binzhou Medical College; 522 Yellow Three \\ Road, Binzhou, Shandong 256603, P. R. China.
}

Received November 28, 2015; accepted January 20, 2016; advance publication released online February 19, 2016

\begin{abstract}
Psoralen is a major active component of Psoralea corylifolia. In the present study, we analyzed psoraleninduced changes in human breast cancer MCF-7/ADR cells and investigated the underlying mechanisms of the anticancer effect on MCF-7/ADR cells. We measured cell viability by 3-(4,5-dimethylthiazol-2-yl)-2,5-diphenyltetrazolium bromide (MTT) assay to evaluate the cytotoxicity and multidrug resistance (MDR) reversal activity of psoralen. The cell cycle distribution and apoptosis, accumulation and efflux of rhodamine123 (Rh123), and P-glycoprotein (P-gp) expression levels of MCF-7/ADR cells treated with psoralen were all detected by flow cytometry (FCM). We assessed P-gp ATPase activity by monitoring ATP consumption. We evaluated the activity of nuclear factor-kappaB (NF- $\kappa \mathrm{B})$ and the expression of E-cadherin, vimentin and $\alpha$-smooth muscle actin (SMA) involved in regulating epithelial-mesenchymal transition (EMT). The results showed that psoralen inhibited the proliferation of MCF-7/ADR cells as shown by G0/G1 phase arrest rather than encouraging apoptosis. It was also observed that psoralen reversed MDR through inhibiting ATPase activity rather than reducing $\mathrm{P}$-gp expression. Our results further showed that psoralen inhibited the migration abilities of MCF-7/ADR cells by repressing EMT possibly through inhibiting the activation of NF- $\kappa$ B. Our findings provided a systematic and detailed description of the anti-cancer effect of psoralen on MCF-7/ ADR cells for the exploration of natural compounds as novel anticancer agents.
\end{abstract}

Key words psoralen; multidrug resistance (MDR); P-glycoprotein (P-gp) ATPase; cell cycle arrest; epithelial-mesenchymal transition (EMT)

The breast cancer incidence in Asia is rising as the leading cause of cancer death among women in the world. It is a systemic disease, with the primary tumor representing the localized form of the disease. At present, the main treatment for breast cancer include chemotherapy, surgery, radiotherapy, hormonal therapy and targeted antibodies. The overall treatment effect is reassuring, but still facing many thorny issues, lacking of specificity and poor avoidance of recurrence. The chemotherapy resistance, the metastasis and recurrence, these are all the reasons for breast cancer treatment failure. Plantderived natural products play a very important role in the area of cancer therapy research as a source of numerous currently used anticancer agents ${ }^{1)}$ including vincristine, vinblastine, paclitaxel, camptothecin derivatives, epipodophyllotoxin, and many others. ${ }^{2)}$ Therefore, there is a great need for continuous search for new antineoplastic agents from plant sources.

In the 1970s, Psoralea corylifolia is widely distributed in Southeastern Asian countries had inspired scientific interest with clinical applications, ${ }^{3)}$ and then it is officially listed in the Pharmacopoeia of the traditional Chinese medicine (TCM) (Edit 2010 Vol. II). As is known to all, two of the main ingredients of furanocoumarins are psoralen and isopsoralen which play a role in cell proliferation and differentiation. Psoralens are photoactive compounds that readily alkylate DNA when activated by longwave ultraviolet light, ${ }^{4)}$ well known in traditional Chinese medicine as "Buguzhisu." It was Thornes that who first proposed the immunomodulatory activity of coumarin and its utility in malignant melanoma, ${ }^{5)}$ then re-

\footnotetext{
${ }^{\#}$ These authors contributed equally to this work.

lated research was made about the photoactivated coumarins in bladder, ${ }^{6)}$ mucoepidermoid carcinoma ${ }^{7)}$ and breast cancer ${ }^{8)}$ with potential for their use in clinical treatments.

It has been proved that the proliferation appeared to be significantly promoted in estrogen receptor (ER) positive MCF-7 cells treated with low concentration of psoralen $(0.01 \mu \mathrm{M})^{9)}$ and inhibited with high concentration. Our previous research also has shown that psoralen promotes proliferation when $<10.75 \mu \mathrm{M}$ and inhibit proliferation when $>21.5 \mu \mathrm{M}$ in a dosedependent manner in MCF-7/ADR cells. There are also studies have suggested that psoralen can inhibit metastasis of breast cancer. ${ }^{10)}$ However the mechanism of anticancer effects of psoralen has heretofore not been deeply considered and is the subject of our research. In addition, chemotherapy or radiotherapy combined with psoralen may take advantage of the synergic effects and consequently reduce the dosage with lower toxicity. ${ }^{11)}$ Therefor the antitumoral activity of psoralen was evaluated in the human breast cancer MCF-7 cells and the multidrug-resistant MCF-7/ADR cells which displayed variations in proliferation and metastasis ability.

\section{MATERIALS AND METHODS}

Chemicals and Materials Psoralen was obtained from Herbest Co., Ltd. (Baoji, China). Cell culture reagents were purchased from GIBCO Life Technology (Grand Island, NY, U.S.A.). Adriamycin and Cell cycle kit was obtained from KeyGen Biotech Co., Ltd. (Nanjing, China). Antibodies specific for the P-glycoprotein (P-gp), and antimouse immunoglobulin $\mathrm{G}(\mathrm{IgG})$, horseradish peroxidase (HRP)-linked anti- 
body were purchased from Abcam (Cambridge, MA, U.S.A.). $\beta$-Actin antibody was obtained from Proteintech (Proteintech Group, CHI, U.S.A.). RevertAid Fist Strand cDNA Synthesis Kit and RealMaster Mix (SYBR-Green) were purchased from Thermo Scientific (Waltham, MA, U.S.A.). 3-(4,5-Dimethylthiazol-2-yl)-2,5-diphenylterazolium bromide (MTT) and TRIzol and Rhodamine123 (Rh123) were obtained from Sigma (St. Louis, MO, U.S.A.). P-gp-Glo assay systems were purchased from Promega Corporation. Fluorescein isothiocyanate (FITC)-labeled Anti-P Glycoprotein antibody UIC2 was purchased from Abcam (ab66250).

Cell Lines MCF-10A, MCF-7 and MCF-7/ADR cells (Nanjing KGI Biological Technology Development Co., Ltd., Nanjing, China).

Cell Culture MCF-10A non-cancerous human mammary epithelial cells were cultured in serum-free mammary epithelial cell growth medium (MEGM) with supplements specified by the manufacturer. MCF-7 and MCF-7/ADR cells were maintained in RPMI-1640 medium supplemented with $10 \%$ fetal bovine serum (FBS), $0.1 \mathrm{mg} / \mathrm{mL}$ penicillin $\mathrm{G}$ and $100 \mathrm{mg} / \mathrm{mL}$ streptomycin in a humidified atmosphere of $5 \%$ $\mathrm{CO}_{2}$ at $37^{\circ} \mathrm{C}$. MCF-7/ADR cells were cultured in the medium containing $1 \mu \mathrm{g} / \mathrm{mL}$ ADR in order to maintain the multidrug resistance (MDR) phenotype, and were then maintained in drug-free medium for at least two days prior to use. The culture medium was changed for every $2 \mathrm{~d}$.

Analysis of Cell Viability The effects of psoralen on cell proliferation were measured by MTT assay. MCF-10A and MCF-7/ADR cells were cultured in 96-well plates at a cell density of $2 \times 10^{4}$ cells per well for $48 \mathrm{~h}$. The medium was then removed and replaced by fresh medium containing different concentrations of psoralen $(0,21.5,43.0,64.5,86.0,107.5 \mu \mathrm{M})$ for $48 \mathrm{~h}$. Cells in the negative control group were incubated with RPMI-1640 culture medium supplemented with $0.1 \%$ dimethyl sulfoxide (DMSO). Cells were incubated with $10 \mu \mathrm{L}$ MTT $(5 \mathrm{mg} / \mathrm{mL})$ for $4 \mathrm{~h}$, and then discarded the medium and added $200 \mu \mathrm{L}$ DMSO. The spectrophotometric absorbance was measured at $490 \mathrm{~nm}$ with enzyme-labeling instrument after the crystals were fully dissolved. Growth inhibition rate (GIR) was calculated as follows GIR $(\%)=\left[\left(\mathrm{OD}_{\text {negative }}\right.\right.$ group $-\mathrm{OD}_{\text {test }}$ group $) /\left(\mathrm{OD}_{\text {negative }}\right.$ group $) \times 100 \%$. The results were presented as the mean \pm standard deviation (S.D.).

Cell Cycle and Apoptosis Assay We evaluated cell cycle distribution and apoptosis by flow cytometry (FCM) analysis. MCF-7/ADR cells $\left(6 \times 10^{4}\right)$ were seeded in 6 -well plates incubated in medium containing $10 \% \mathrm{FBS}$ and psoralen $(0$, $21.5,43.0,64.5,86.0,107.5 \mu \mathrm{M})$ for 24 and $48 \mathrm{~h}$ at $37^{\circ} \mathrm{C}$. Cellular DNA content of $1 \times 10^{4}$ cells from each sample was determined by Cell cycle kit (KeyGen Biotech Co., Ltd.). Cell cycle phase distribution was analyzed with the ModFit LT 3.2 software. The cell apoptosis was analyzed by AnnexinV-FITC/ propidium iodide (PI) apoptosis detection kit. Finally, samples were analyzed by BD fluorescent activated cell sorter (FACS) Calibur (BD Bioscience U.S.A.).

MDR Reversal Activity $\mathrm{The} \mathrm{IC}_{50}$ of MCF-7/ADR cells treated with psoralen at a concentration of $43.0 \mu \mathrm{M}$ was analyzed by the same MTT method described above. The reversal index $(\mathrm{RI})=\mathrm{MCF}-7 / \mathrm{ADR} \mathrm{IC}_{50}$ treated with $\mathrm{ADR} / \mathrm{MCF}-7 / \mathrm{ADR}$ $\mathrm{IC}_{50}$ treated with $\mathrm{ADR}$ and psoralen $(43.0 \mu \mathrm{M})$. The experiment was repeated three times, each time with three parallel samples.
Effects on Rh123 Accumulation We analysed the cellular accumulation of Rh123 which is the specificity substrate of P-gp by FCM according to standard procedures. ${ }^{12)}$ The cells treated with $43.0 \mu \mathrm{M}$ of psoralen were cultured at $37^{\circ} \mathrm{C}$ for $3 \mathrm{~h}$, and then incubated for $0.5 \mathrm{~h}$ with $5 \mu \mathrm{g} / \mathrm{mL}$ Rh123. The cells were harvested and washed three times with cold phosphate buffered saline (PBS). The fluorescence intensity was analyzed by BD FACS Calibur (BD Bioscience U.S.A.).

Flow Cytometric Analysis of Cell P-gp Expression Level FITC-labeled Anti-P Glycoprotein antibody UIC2 (ab66250) was purchased from Abcam. MCF-7, MCF-7/ADR and MCF-7/ ADR + psoralen cells $\left(1 \times 10^{6}\right)$ were respectively added $100 \mu \mathrm{L}$ warm UIC2 binding buffer PBS, $1 \%$ bovine serum albumin (BSA)), incubated $10 \mathrm{~min}$ at $37^{\circ} \mathrm{C}$, then added $10 \mu \mathrm{L}$ FITClabeled UIC2 incubating $30 \mathrm{~min}$. The cells were harvested and resuspended in $500 \mu \mathrm{L}$ cold UIC2 binding buffer, and read the FITC fluorescence using a BD FACS Calibur.

ATPase Activity We determined the ATPase activity by P-gp-Glo Assay Systems (Promega, U.S.A.). Sodium vanadate $\left(\mathrm{Na}_{3} \mathrm{VO}_{4}\right)$ was used as a P-gp ATPase inhibitor. Verapamil (Ver) served here as a positive control for drug stimulation of P-gp ATPase activity. The activity of P-gp ATPase was measured after treated with psoralen. The luminescence of samples is negatively correlated with the P-gp ATPase activity. The relative light units (RLU) of the untreated samples (NT) compared to the RLU of $\mathrm{Na}_{3} \mathrm{VO}_{4}$-treated samples reflects the consumption of ATP by the basal P-gp ATPase activity and is expressed as $\triangle R L U_{\text {basal }}$. The RLU in the psoralen treated samples compared to the $\mathrm{Na}_{3} \mathrm{VO}_{4}$-treated samples reflect the consumption of P-gp ATPase activity in the presence of psoralen and is expressed as $\triangle \mathrm{RLU}_{\mathrm{TC}}$. The activity ratio $\left(\Delta \mathrm{RLU}_{\mathrm{TC}} / \Delta \mathrm{RLU} \mathrm{U}_{\text {basal }}\right)$ reflects the effect of spiraled treatment on ATPase activity.

Cell Migration Assay Cell scarification is one of the best methods to determine the motion characteristics of tumor cells. MCF-7/ADR cells were cultured in 6 well plates for $12 \mathrm{~h}$ with serum-free medium to reduce the influence of cell proliferation ( 3 wells per group). After scraping the cell monolayer with a sterile micropipette tip, then cultured with psoralen $(43.0 \mu \mathrm{M})$. The cell number in the scraped zone of each dish was counted at $40 \times$ magnification after scraping $(0,24,48 \mathrm{~h})$. The changes in migration measured by Image-Pro plus 6.0 software were determined by comparing the difference in wound-healing areas after $48 \mathrm{~h}$.

Real-Time Polymerase Chain Reaction (PCR) To validate whether psoralen reduces MCF-7/ADR cells metastasis via down-regulating EMT, the expression levels of EMT markers were detected by real-time PCR. The primers used were as follows: E-cadherin (forward: 5'-CCA CCC TGGCTT TGA CGC-3'， reverse: 5'-AGG TGGAGT CCC AGGCGT AG-3'), vimentin (VIM) (forward: 5'-TCA GAC AGG ATG TTG ACA AT-3', reverse: 5'-GACATG CTG TTC CTGAATCT- $\left.3^{\prime}\right), \alpha$-Smooth Muscle Actin ( $\alpha$-SMA) (forward: 5'-GAT GTA CCC TGG GAT CGC TGA C-3', reverse: $5^{\prime}$-AAGCAT TTGCGGTGGACGAT-3') and $\beta$-actin (forward: 5'-CCT GGC ACC CAGCAC AAT-3'， reverse: 5'-GGG CCGGACTCG TCA TAC-3'). The real-time PCR reaction was performed with the SYBR green detection system (Thermo Scientific, Waltham, MA, U.S.A.). $\beta$-Actin served as an endogenous control. All cycle threshold $(\mathrm{Ct})$ values were determined in real time using CFX96 ${ }^{\mathrm{TM}}$ Real-Time PCR Detection 
(Bio-Rad, CA, U.S.A.). The data were analyzed by $2^{-\Delta \Delta C t}$.

Immunofluorescence and Western Blot Analysis To further explore the molecular mechanisms responsible for psoralen mediated inhibition of EMT, we focused on NF- $\kappa \mathrm{B}$ signaling because activation of NF- $\kappa \mathrm{B}$ is a central feature of EMT and psoralen has been demonstrated as inhibitors of $\mathrm{NF}-\kappa \mathrm{B} / \mathrm{DNA}$ interaction to inhibit tumor invasion and migration. ${ }^{13)}$ The nucleus NF- $\kappa \mathrm{B}$ expression was detected in MCF-7/ ADR and MCF-7/ADR+psoralen cells by immunofluorescence and Western blot analysis. The nuclear protein from MCF-7/ $\mathrm{ADR}$ and $\mathrm{MCF}-7 / \mathrm{ADR}+$ psoralen cells were extracted by the nuclear extract kit (KeyGen Biotech Co., Ltd., Najing, China). Western blot analysis was conducted according to a standard protocol. ${ }^{14)}$ Image J software was used for densitometric analysis of the bands and all values were normalized to $\beta$-actin.

Statistical Analysis In the presented study, one-way ANOVA was used for analyzing the dose-dependent effect of psoralen on MCF-10A, MCF-7 and MCF-7/ADR cells, and Student's $t$ test was used for analyzing difference between groups. Both one-way ANOVA and Student's t test were performed by using the SPSS 17.0 software (SPSS Inc., Chicago, IL, U.S.A.) and $p<0.05$ was considered significant.

\section{RESULTS}

Effects of Psoralen on the Cell Proliferation Psoralen was demonstrated to inhibit the proliferation of MCF-7/ADR cells in a dose-dependent manner but no such significant effect on MCF-10A cells (Fig. 1). With the increase in concentration, the proliferation of MCF-7/ADR cells gradually
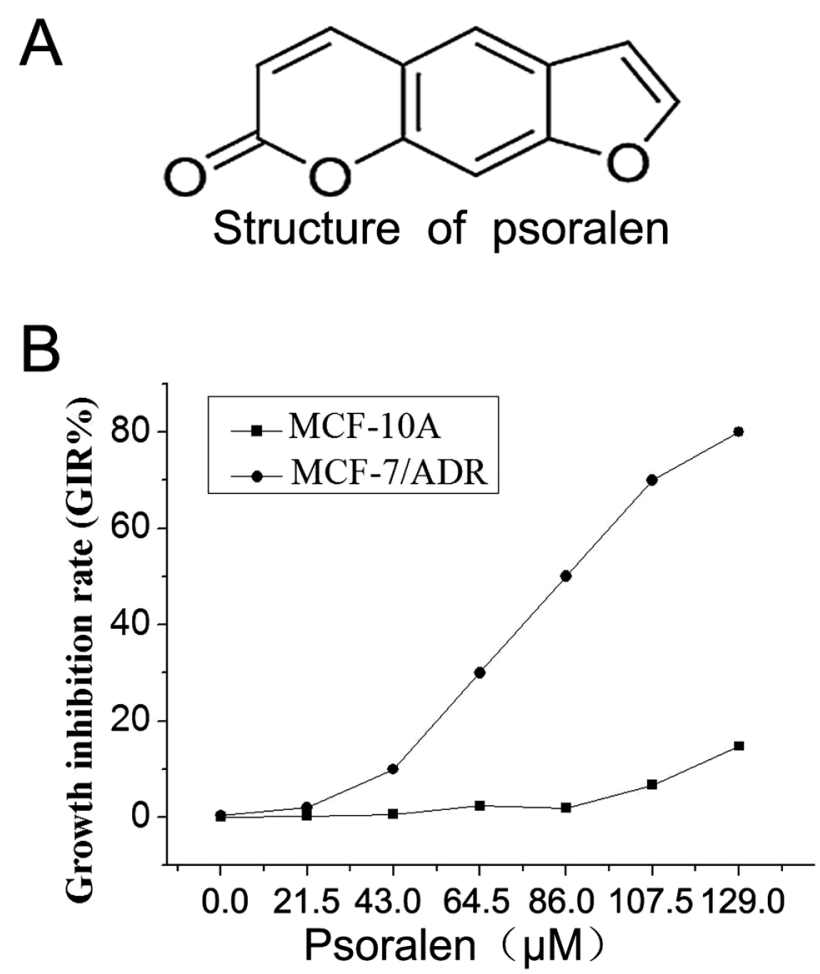

Fig. 1. (A) Structure of Psoralen; (B) Psoralen Inhibited the Proliferation of MCF-7/ADR Cells in a Dose-Dependent Manner but No Such Significant Effect on MCF-10A Cells

The concentration of $43.0 \mu \mathrm{M}$ with inhibitory rate at $10 \%$ was considered to be a non-cytotoxic dose chosen as the working concentration in the subsequent experiments. Values are the mean \pm S.D. of three experiments. decreased. Psoralen at $43.0 \mu \mathrm{M}$ exhibited the inhibitory rate at $10 \%$ on MCF-7/ADR cells, therefore, the concentration of $43.0 \mu \mathrm{M}$ was considered to be a non-cytotoxic dose chosen as the working concentration in the subsequent experiments. The MDR reversal and the motility and invasive ability effect of psoralen at a concentration of $43.0 \mu \mathrm{M}$ on the MCF-7/ADR cells were investigated.

Psoralen Induces Cell G0/G1 Phase Arrest in MCF-7/ ADR Cells To understand if psoralen inhibited MCF-7/ ADR cells proliferation, we analyzed the effect of psoralen treatment for 24 and $48 \mathrm{~h}$ on cell cycle profile and apoptosis in MCF-7/ADR cells by FCM (Fig. 2). Percentage of subpopulation of cells after psoralen treatmented $48 \mathrm{~h}$ in cell cycle phases G0/G1, S, and G2/M are shown in Fig. 2A. The population in cell cycle phase G0/G1 in psoralen treated cells was statistically increased compared to those in control cells in a concentration-dependent manner after psoralen treatmented 24h (Fig. 2B) and 48h (Fig. 2C), but has no effect on cell apoptosis $(p>0.05)$.

Psoralen Decreases Drug Efflux and Enhances Cytotoxicity by Inhibiting the P-gp ATPase Activity The chemosensitivity of MDR cells MCF-7/ADR to the chemotherapy drugs was measured by comparing $\mathrm{IC}_{50}$ values. The reversal fold value $=\left(\mathrm{IC}_{50}\right.$ of $\mathrm{MCF}-7 / \mathrm{ADR}+$ psoralen $/ \mathrm{IC}_{50}$ of MCF-7/ADR). The result showed that the $\mathrm{IC}_{50}$ of MCF-7/ $\mathrm{ADR}+$ psoralen and MCF-7/ADR were $25.59 \pm 1.74 \mu \mathrm{g} / \mathrm{mL}$ and $86.91 \pm 3.61 \mu \mathrm{g} / \mathrm{mL}$. The reversal fold value was 3.39 fold when the cells were treated with $8 \mu \mathrm{g} / \mathrm{mL}$ psoralen, indicating that psoralen significantly reverses MDR and increases cytotoxicity of ADR to MCF-7/ADR cells (Fig. 3A) $(* p<0.05)$. We hypothesized that psoralen reversal drug resistance through reducing the expression of MDR1 or inhibiting drug efflux from MCF-7/ADR cells. We therefore examined the efflux capacity using Rh123 dye. The accumulation of Rh123 was measured by the fluorescent intensity. The percentage of remaining intracellular Rh123 in MCF-7/ADR+psoralen is $59.92 \pm 3.16 \%$ (relative fluorescence intensity) and significantly higher than the control group $22.54 \pm 1.32 \%$ (Fig. 3B) $(* p<0.01)$. The FCM assay results showed that the expression of P-gp between MCF-7/ADR+ psoralen and MCF-7/ADR groups had no significant differences (Fig. 3C). Then we detected the P-gp ATPase activity and found that the $\Delta \mathrm{RLU}_{\mathrm{TC}(\mathrm{pso})}=2.03 \pm 0.72 \times 10^{4}$, $\Delta \mathrm{RLU}_{\mathrm{TC}(\mathrm{Ver})}=2.93 \pm 0.41 \times 10^{4}, \Delta \mathrm{RLU}_{\mathrm{TC}(\mathrm{pso}+\mathrm{Ver})}=5.07 \pm 0.14 \times 10^{4}$ and the $\Delta \mathrm{RLU}_{\text {basal }}=2.43 \pm 0.25 \times 10^{4}$, indicating that psoralen significantly decreased ATPase activity of P-gp $(* p<0.05)$ but enhanced the verapamil-stimulated ATPase activity $\left({ }^{\#} p<0.05\right)$, which indicating that there may be a synergistic effect of psoralen and verapamil on P-gp ATPase activity (Fig. 3D).

Effects of Psoralen on the Migration Ability of MCF-7/ ADR Cells The effect of psoralen on MCF-7/ADR cells migration was determined by the wound-healing assay. As shown in Fig. 4A, the MCF-7/ADR cells exposed to $43.0 \mu \mathrm{M}$ of psoralen increase slowly in the denuded zone under light microscopy as compared to the untreated cells after 24 and $48 \mathrm{~h}$. The quantitative data in Fig. 4B revealed that psoralen could inhibit the migration of MCF-7/ADR cells $(* p<0.05)$.

Psoralen Reduces EMT in MCF-7/ADR Cells The morphology of MCF-7/ADR cells treated with psoralen was observed. The untreated MCF-7/ADR cells retained their elongated spindle-like morphology with sharp borders. However, MCF-7/ADR cells treated with psoralen displayed a cobble- 
A

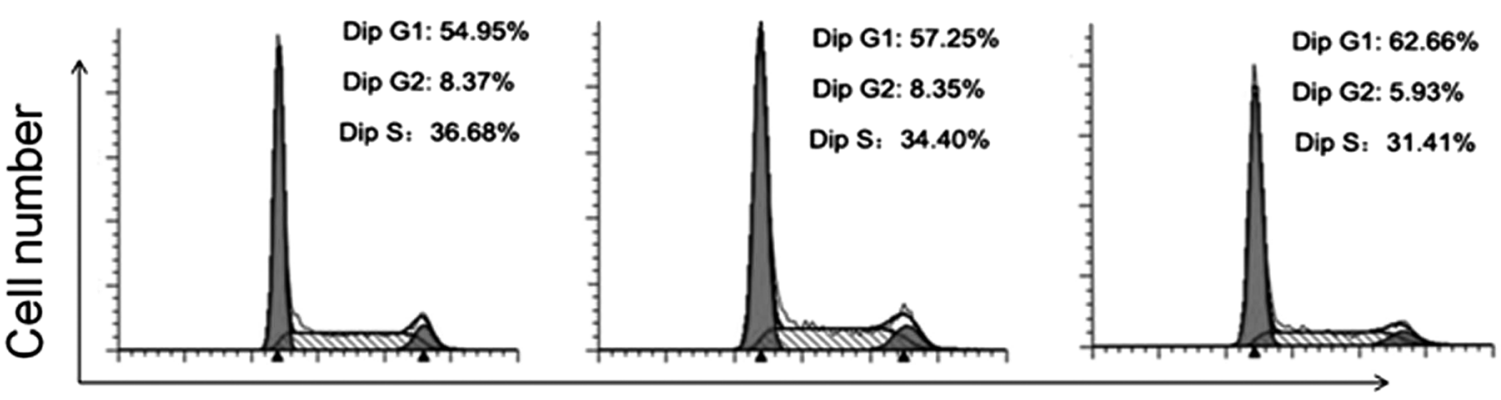

$48 \mathrm{~h}$

DNA content

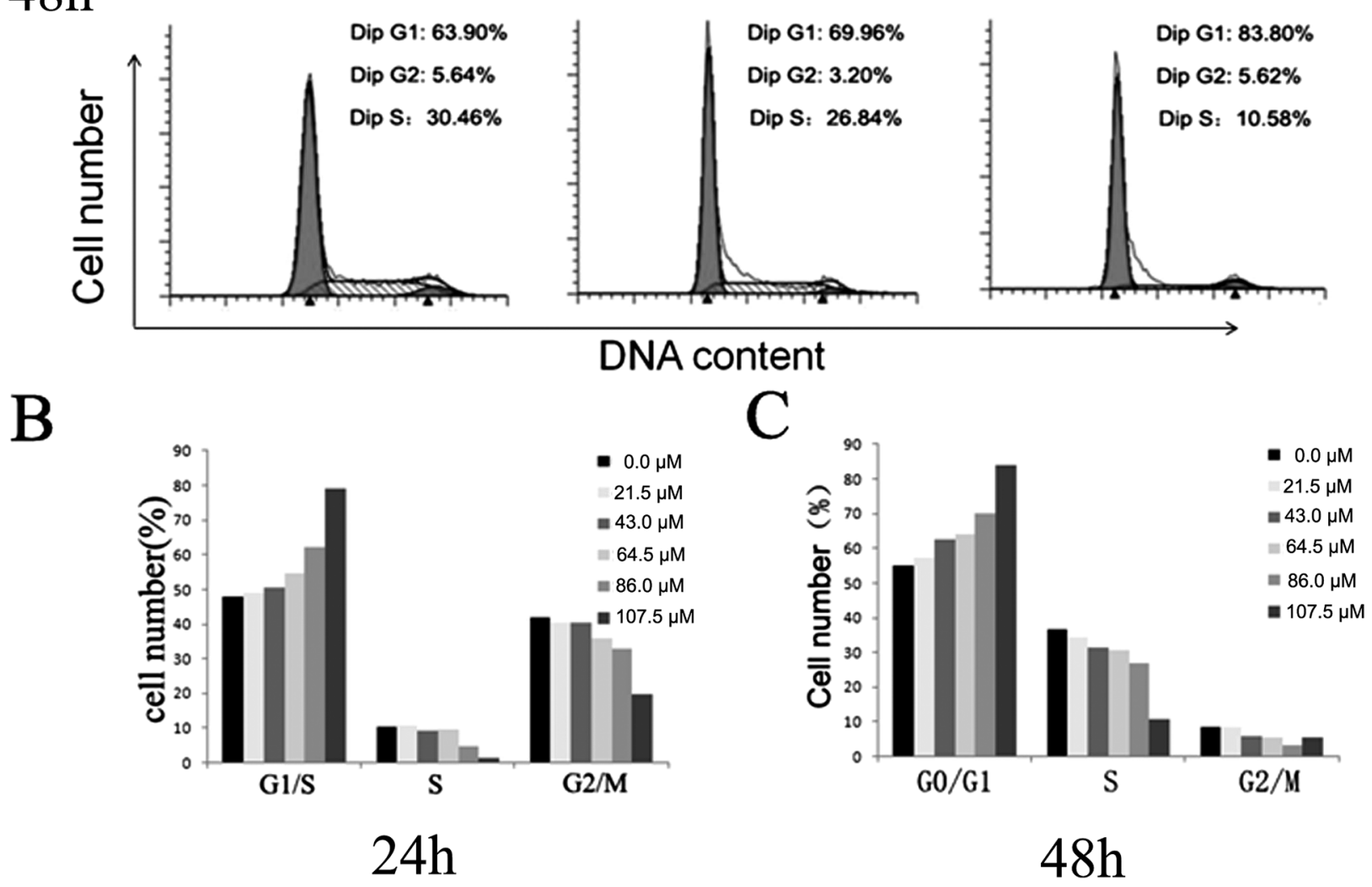

Fig. 2. The Effects of Psoralen on Cell Cycle Profile of G0/G1, S, and G2/M Phases

Psoralen treatment increased the percentage of G0/G1 phase in a concentration-dependent and time-dependent manner for 24 and $48 \mathrm{~h}$, but has no effect on cell apoptosis $(p>0.05)$

stone-like epithelial phenotype, grew as a monolayer and formed islands of grouped cells with tight cell-cell contact (Fig. 5A). To validate whether psoralen reduces MCF-7/ADR cells metastasis via down-regulating EMT, the expression levels of EMT markers were detected by real-time PCR. We found that the epithelial marker, E-cadherin was dramatically up-regulated in the psoralen treated MCF-7/ADR cells, but mesenchymal markers such as VIM and $\alpha$-SMA were strongly down-regulated in psoralen treated MCF-7/ADR cells (Fig. $5 \mathrm{~B})$. These data suggest that psoralen might be a negative mediator of EMT and metastasis in MCF-7/ADR cells.

Psoralen Represses Cell EMT by Inhibiting the Activation of the NF- $\boldsymbol{k}$ B Pathway The NF- $\kappa$ B activation was investigated by immunofluorescence and Western blotting. The data in Fig. 6A demonstrated that psoralen inhibited the nuclear translocation of NF- $\kappa \mathrm{B}$ p 65 , with a decreased nuclear level, and this effect was confirmed by Western blotting (Fig. $6 \mathrm{~B})$. The nucleus NF- $\kappa \mathrm{B}$ expression was significantly downregulated in $\mathrm{MCF}-7 / \mathrm{ADR}+$ psoralen cells $(* p<0.05)$ (Fig. 6C).
These results suggested that psoralen suppressed cell EMT possibly by inhibiting NF- $\kappa \mathrm{B}$ activation which is required in EMT. ${ }^{15,16)}$

\section{DISCUSSION}

The aim of this study was to characterize the anti-cancer effect of psoralen on MCF-7/ADR cells. Several lines of evidence indicate that psoralen mediates a variety of cell processes including cell death, proliferation, inflammation and migration. In the past few years, psoralen has been characterized as a tumor suppressor in various tumors. In the present study, we provided a systematic and detailed description of the anti-cancer effect of psoralen on human MCF-7/ADR cells for the first time. Our results suggest that the potent anti-cancer activity of psoralen correlates with the inhibition of cell proliferation, retardation of cell cycle, reversal of MDR and suppression of cell invasion and migration.

Here, we found that psoralen decreased cells viability in a 

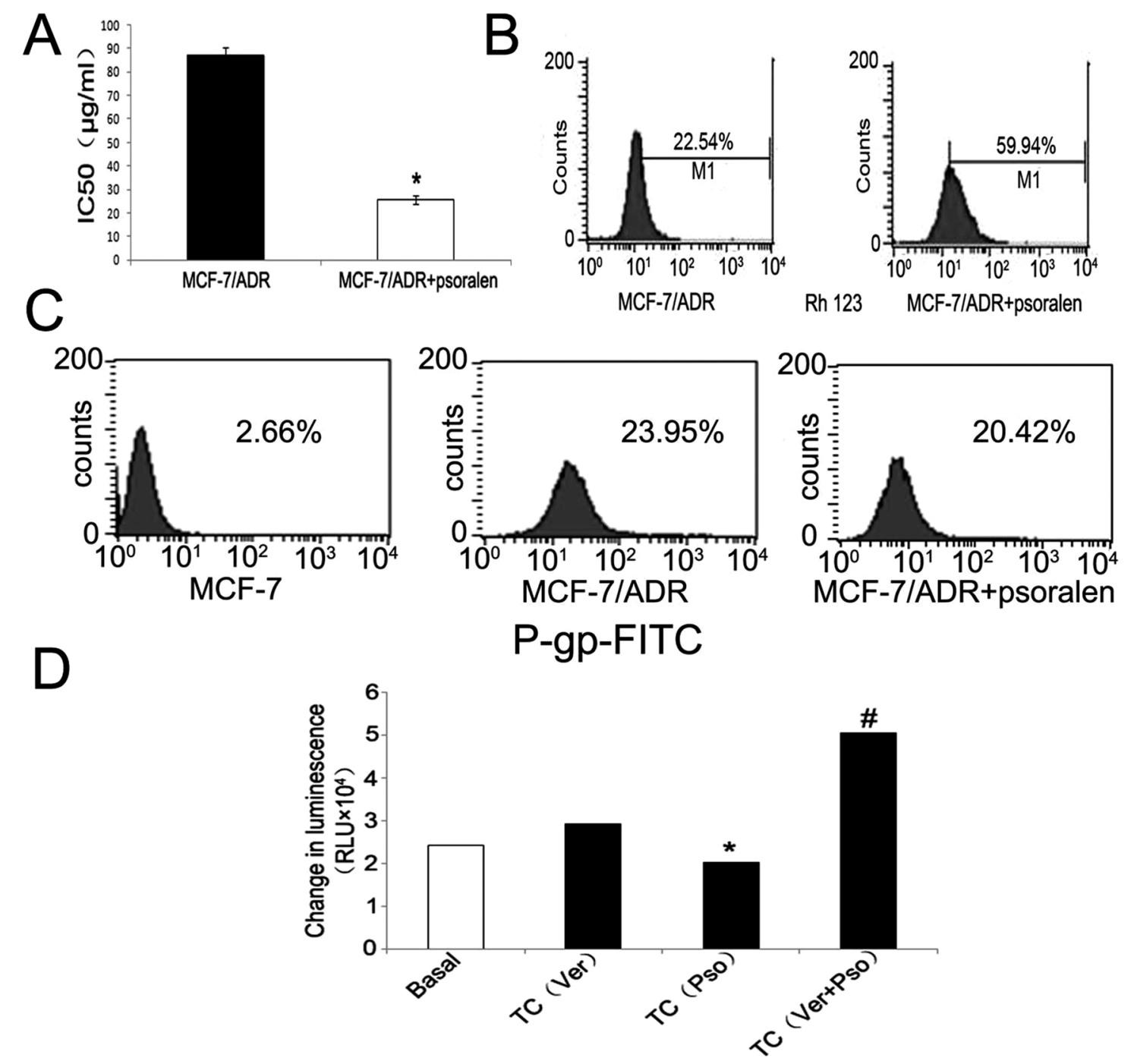

Fig. 3. Psoralen Decreases Drug Efflux and Enhances Cytotoxicity by Inhibiting the P-gp ATPase Activity

(A) The chemosensitivity of MDR cells MCF-7/ADR to the chemotherapy drugs was measured by comparing $\mathrm{IC}_{50}$ values $(* p<0.05)$. (B) The efflux capacity was detected using Rh123 dye. The accumulation of Rh123 was measured by the fluorescent intensity. The percentage of remaining intracellular Rh123 in MCF-7/ADR treated with psoralen significantly higher than the control group $\left({ }^{*} p<0.01\right)$. (C) The flow cytometry assay results showed that the expression of P-gp between MCF-7/ADR+psoralen and MCF-7/ADR groups had no significant differences. (D) P-gp ATPase assay was conducted. Psoralen significantly decreased ATPase activity of P-gp $(* p<0.05)$ but enhanced the verapamil-stimulated ATPase activity $(* p<0.05)$.

dose-dependent manner. To mechanistically explain our observation, we investigated psoralen effects on cell cycle and apoptosis. We found that psoralen prevented cell progression from $\mathrm{G} 1$ into $\mathrm{S}$ phase and blocked cell proliferation which is consistent with previous studies conducted in melanoma cells and other cell lines. ${ }^{17,18)}$ In fact, previous study reported that psoralen arrested HaCaT cells in the $\mathrm{S}$ phase of the cell cycle, which suggested psoralen had potential selective cell cycle arrest ability. However, there was no significant difference or even a slight decrease of apoptosis cells in MCF-7/ADR cells after treated with psoralen for $48 \mathrm{~h}$. These results suggested that psoralen inhibited MCF-7/ADR cells proliferation by blocking cell cycle but not apoptosis which was different from other studies ${ }^{19)}$ finding that psoralens induced KB cells death mediated by cell apoptosis. The differences of apoptotic response to psoralen may suggest the cell type specific.

In this study, we utilized MCF-7 and drug-selected P-gp over expressing MCF-7/ADR cells to evaluate the reversal effect of psoralen on P-gp-mediated MDR. As demonstrated, psoralen significantly increased the intracellular accumulation of Rho123 in P-gp-overexpressing MCF-7/ADR cells which were consistent with the adriamycin cytotoxic data. The results showed P-gp-mediated MDR can be reversed by inhibiting its function, because the expression of MDR1 mRNA or P-gp was not changed. Thus, we propose that the direct interference with P-gp function may contribute to the P-gp ATPase. The results showed that psoralen separately inhibited the P-gp ATPase activity but enhanced the verapamil-stimulated ATPase activity indicating that psoralen and verapamil would have a synergistic effect on P-gp ATPase activity and the binding position maybe partially overlaped. And also, more complicated mechanisms still require further study by obtaining co-crystal structures of P-gp to explain the involving functional modulation of psoralen. ${ }^{20}$

In recent years, many studies have found that MCF-7/ ADR with over-expression of P-gp showed stronger invasive ability and malignant potential compared to parental control MCF-7 cell possibly related to the loss of E-cadherin expression which is the epithelial marker. ${ }^{21)}$ This process is essentially dependent on the prominent biological event referred 


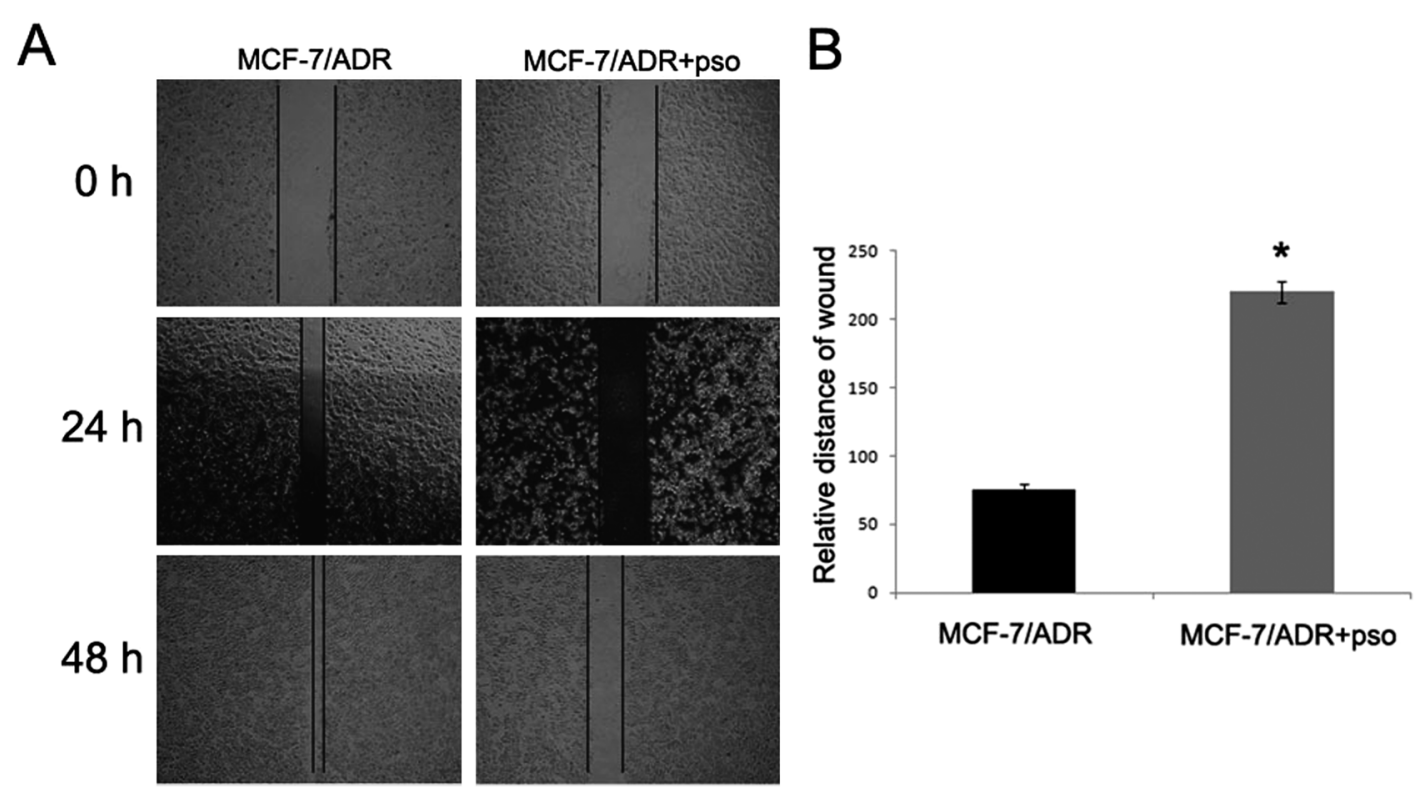

Fig. 4. Psoralen Inhibits Migration Ability of MCF-7/ADR Cells

(A) Movement ability of MCF-7/ADR cells and MCF-7/ADR+psoralen cells was detected by scratch wound healing assays. The MCF-7/ADR cells exposed to 43.0 $\mu \mathrm{M}$ of psoralen increase slowly in the denuded zone under light microscopy as compared to the untreated cells after 24 and $48 \mathrm{~h}$. (B) By wound distance analysis, the movement ability of MCF-7/ADR cells was significantly inhibited by psoralen $(* p<0.05)$.

A

MCF-7

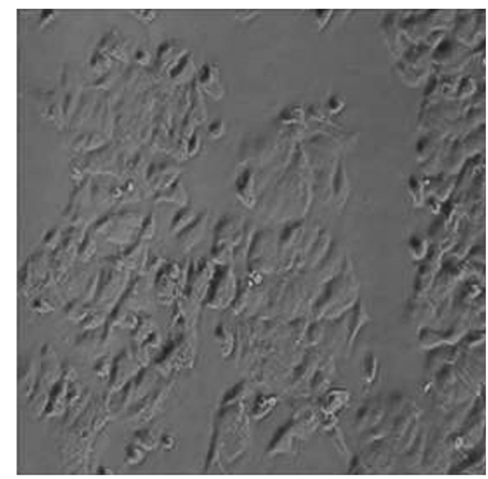

MCF-7/ADR

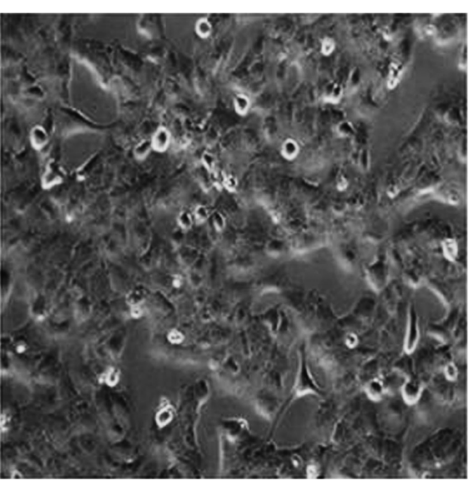

MCF-7/ADR+psoralen

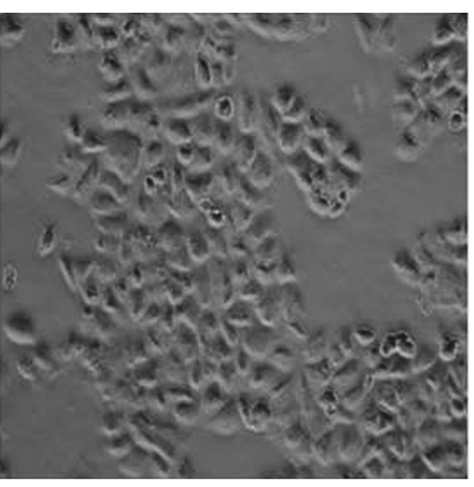

B

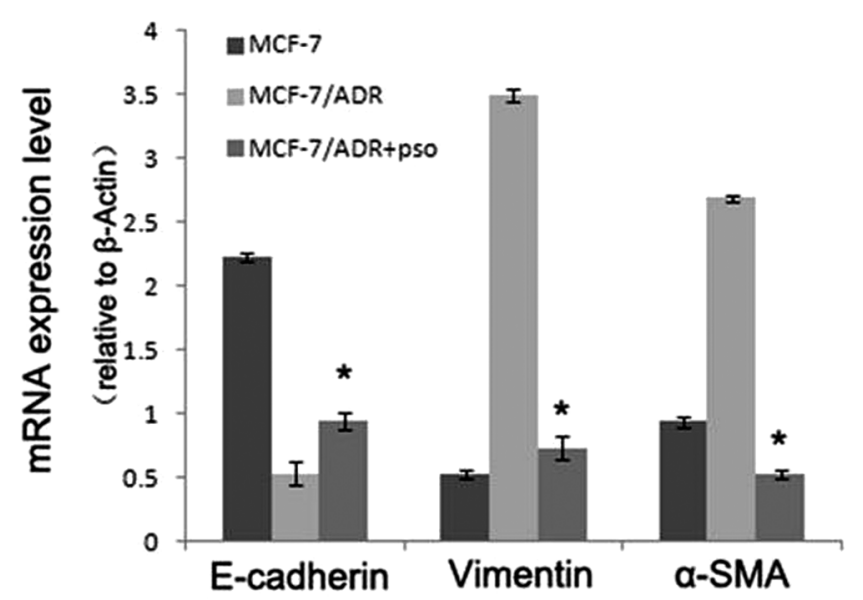

Fig. 5. Psoralen Prevented EMT in MCF-7/ADR Cells

(A) The morphology of MCF-7/ADR cells treated with psoralen was observed $(\times 200)$. MCF-7/ADR cells treated with psoralen displayed a cobblestone-like epithelial phenotype with tight cell-cell contact. (B) mRNA levels of epithelial markers (E-cadherin) was dramatically up-regulated and mesenchymal markers (VIM and $\alpha$-SMA) were strongly down-regulated in the psoralen treated MCF-7/ADR cells $(* p<0.05)$. 

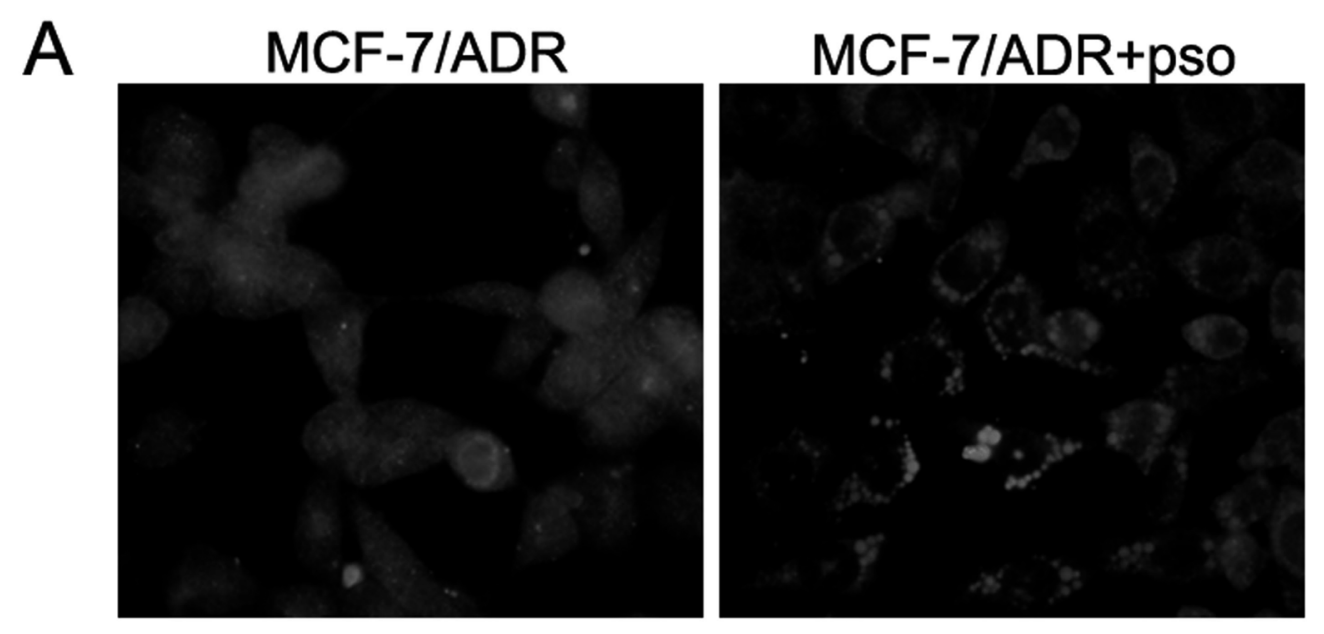
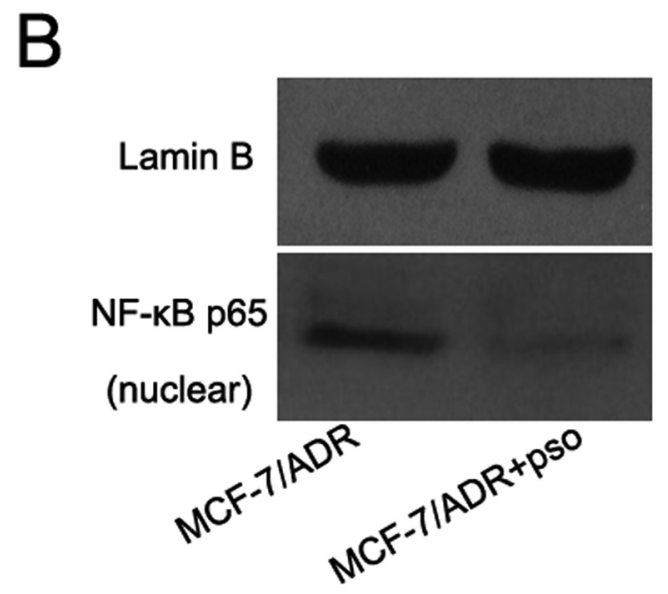
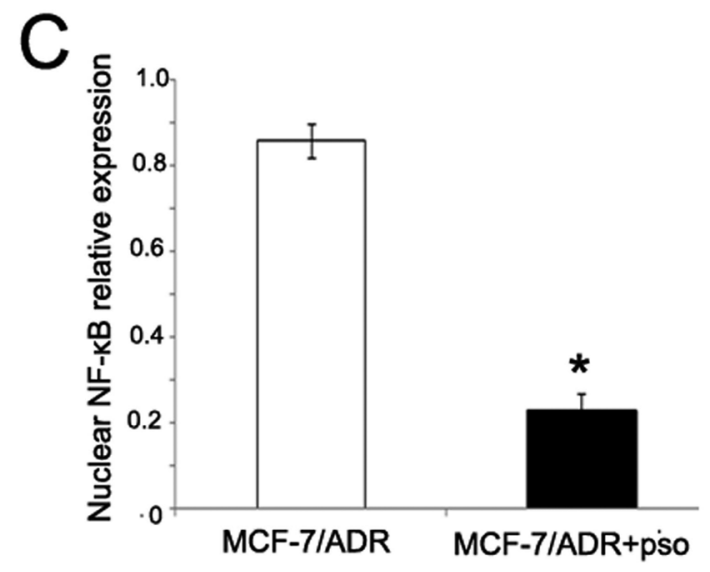

Fig. 6. The Activation of the NF- $\kappa$ B Pathway

(A) Immunofluorescent staining of NF- $\kappa \mathrm{B}$ p65 in MCF-7/ADR cells $(\times 200)$. The NF- $\kappa \mathrm{B}$ expression of the MCF-7/ADR cells was mainly in the cell nucleus, while the activation of NF- $\kappa$ B blocked by psoralen was mainly in the circum-nucleus. (B) Western blotting demonstrated that the nucleus NF- $\kappa$ B expression was significantly downregulated in MCF-7/ADR+psoralen cells. (C) Values were expressed as the mean \pm S.D. $* p<0.05$ vs. control group.

to as $\mathrm{EMT}^{22,23)}$ which enables cells of epithelial phenotype to generate mesenchymal derivatives. Therefore, targeting EMT may serve as an efficient strategy for the treatment of malignant and metastatic tumors. The previous studies showed that psoralen influence extracellular matrix (ECM) which played an important role in the proliferation and metastasis of tumors. ${ }^{24)}$ In the present study, we used Transwell/Matrigel invasion assays to demonstrate that psoralen inhibitory effect on MCF-7/ADR cells invasion, then detected the morphology and the expression levels of EMT markers. These data suggest that psoralen might be a negative mediator of EMT and metastasis in MCF-7/ADR cells. Concomitantly we found that psoralen inhibits NF- $\kappa \mathrm{B}$ activation which is required in EMT. One explanation for this complex behavior is through contextual effects of NF- $\kappa$ B associated modulators. There are several studies have shown that psoralen derivatives as inhibitors of $\mathrm{NF}-\kappa \mathrm{B}$ pathway ${ }^{25,26)}$ inhibit cells EMT and metastasis. Therefore more work will be required to identify whether psoralen inhibited EMT via the NF- $\kappa$ B pathway.

In conclusion, the antitumoral activity of psoralen was evaluated on human MCF-7/ADR cells in proliferation, MDR and metastasis ability and provided molecular basis for the further development of natural compounds as novel anticancer agents for human breast cancer. The natural products combined with the traditional chemotherapeutic agents may take advantage of the synergic effects and consequently decrease the side effects at the systemic level, providing a new opportunity for the treatment of breast tumors.

Acknowledgments This work was supported by National Natural Science Foundation of China (No. 81173601) and Scientific Research Staring Foundation of Binzhou Medical College (No. BY2014KYQD36).

Conflict of Interest The authors declare no conflict of interest.

\section{REFERENCES}

1) Ramirez-Mares MV, Chandra S, de Mejia EG. In vitro chemopreventive activity of Camellia sinensis, Ilex paraguariensis and $\mathrm{Ar}$ disia compressa tea extracts and selected polyphenols. Mutat. Res., 554, 53-65 (2004).

2) Cragg GM, Newman DJ. Plants as a source of anti-cancer agents. $J$. Ethnopharmacol., 100, 72-79 (2005).

3) Gaind KN, Dar RN, Kaul RN. Antistaphylococcal activity of seeds of Psoralea corylifolia. J. Pharm. Sci., 53, 1428-1429 (1964).

4) Saffran WA, Ahmed A, Binyaminov O, Gonzalez C, Gupta A, Fajardo MA, Kishun D, Nandram A, Reyes K, Scalercio K, Senior $\mathrm{CW}$. Induction of direct repeat recombination by psoralen-DNA adducts in Saccharomyces cerevisiae: defects in DNA repair increase 
gene copy number variation. DNA Repair, 21, 87-96 (2014).

5) Thornes RD, Lynch G, Sheehan MV. Cimetidine and coumarin therapy of melanoma. Lancet, 320, 328 (1982).

6) Keane TE, Petros JA, Velimirovich B, Yue KT, Graham SJ Jr. Methoxypsoralen phototherapy of transitional cell carcinoma. Urology, 44, 842-846 (1994).

7) Wu JZ, Situ ZQ, Wang W, Chen JY, Liu B. Antitumor activity of psoralen on mucoepidermoid carcinoma cell line MEC-1. Chin. Med. J., 105, 913-917 (1992).

8) Wu S, Zhang Z, Zhao J. An experimental study on antitumor activity of psoralen on mammary cancer cell line EMT6 in vitro and in vivo. Zhongguo Zhong Yao Za Zhi, 23, 303-305 (1998).

9) Shen LX, Dong XH, Li W, Wang JF, Niu JZ. Effect of quercetin and psoralen on proliferation in MCF-7 cells. Zhongguo Yaolixue Tongbao, 25, 601-605 (2009).

10) Sheng $\mathrm{L}, \mathrm{Wu} \mathrm{CY}$, Chen $\mathrm{XF}$. Inhibitory acting mechanism of psoralen-osthole on bone metastasis of breast cancer-an expatiation viewing from OPG/RANKL/RANK system. Zhongguo Zhong Xi Yi Jie He Za Zhi, 31, 684-689 (2011).

11) Li YG, Hou J, Li SY, Lv X, Ning J, Wang P, Liu ZM, Ge GB, Ren JY, Yang L. Fructus Psoraleae contains natural compounds with potent inhibitory effects towards human carboxylesterase 2. Fitoterapia, 101, 99-106 (2015).

12) Xiang QF, Zhang DM, Wang JN, Zhang HW, Zheng ZY, Yu DC, Li YJ, Xu J, Chen YJ, Shang CZ. Cabozantinib reverses multidrug resistance of human hepatoma HepG2/adr cells by modulating the function of P-glycoprotein. Liver Int., 35, 1010-1023 (2015).

13) Marzaro G, Guiotto A, Borgatti M, Finotti A, Gambari R, Breveglieri G, Chilin A. Psoralen derivatives as inhibitors of NF-kappaB/ DNA interaction: synthesis, molecular modeling, 3D-QSAR, and biological evaluation. J. Med. Chem., 56, 1830-1842 (2013).

14) Wang XH, Ding XM, Li Y, Liu HB, Xue WJ, Tian XH, Feng XS, Jiao FM, Zheng J. Simultaneous blockade of the CD40/CD40L and NF-kappaB pathways prolonged islet allograft survival. Transpl. Int., 25, 118-126 (2012).

15) Tian B, Li X, Kalita M, Widen SG, Yang J, Bhavnani SK, Dang B, Kudlicki A, Sinha M, Kong F, Wood TG, Luxon BA, Brasier AR. Analysis of the TGFbeta-induced program in primary airway epithelial cells shows essential role of NF-kappaB/RelA signaling network in type II epithelial mesenchymal transition. BMC Genom- ics, 16, 529 (2015).

16) Tobar N, Villar V, Santibanez JF. ROS-NF $\kappa$ B mediates TGF- $\beta 1$ induced expression of urokinase-type plasminogen activator, matrix metalloproteinase-9 and cell invasion. Mol. Cell. Biochem., 340, 195-202 (2010).

17) Varga JM, Wiesehahn G, Bartholomew JC, Hearst JE. Dose-related effects of psoralen and ultraviolet light on the cell cycle of murine melanoma cells. Cancer Res., 42, 2223-2226 (1982).

18) Joerges C, Kuntze I, Herzinge T. Induction of a caffeine-sensitive S-phase cell cycle checkpoint by psoralen plus ultraviolet A radiation. Oncogene, 22, 6119-6128 (2003).

19) Wang Y, Hong C, Zhou C, Xu D, Qu HB. Screening antitumor compounds psoralen and isopsoralen from Psoralea corylifolia L. seeds. Evid. Based Complement. Alternat. Med., 2011, 363052 (2011).

20) Xiang Q, Chen W, Ren M, Wang J, Zhang H, Deng DY, Zhang L, Shang C, Chen Y. Cabozantinib suppresses tumor growth and metastasis in hepatocellular carcinoma by a dual blockade of VEGFR2 and MET. Clin. Cancer Res., 20, 2959-2970 (2014).

21) Lu L, Zhou D, Jiang X, Song K, Li K, Ding W. Loss of E-cadherin in multidrug resistant breast cancer cell line MCF-7/Adr: possible implication in the enhanced invasive ability. Eur. Rev. Med. Pharmacol. Sci., 16, 1271-1279 (2012).

22) Chaffer CL, Weinberg RA. A perspective on cancer cell metastasis. Science, 331, 1559-1564 (2011).

23) Fidler IJ. The pathogenesis of cancer metastasis: the 'seed and soil' hypothesis revisited. Nat. Rev. Cancer, 3, 453-458 (2003).

24) Xu K, Pan X, Sun Y, Xu W, Njunge L, Yang L. Psoralen activates cartilaginous cellular functions of rat chondrocytes in vitro. Pharm. Biol., 53, 1010-1015 (2015).

25) Marzaro G, Guiotto A, Borgatti M, Finotti A, Gambari R, Breveglieri G, Chilin A. Psoralen derivatives as inhibitors of NF-kappaB/ DNA interaction: synthesis, molecular modeling, 3D-QSAR, and biological evaluation. J. Med. Chem., 56, 1830-1842 (2013).

26) Li J, Deng Z, Wang Z, Wang D, Zhang L, Su Q, Lai Y, Li B, Luo Z, Chen X, Chen Y, Huang X, Ma J, Wang W, Bi J, Guan X. Zipper-interacting protein kinase promotes epithelial-mesenchymal transition, invasion and metastasis through AKT and NF- $\kappa$ B signaling and is associated with metastasis and poor prognosis in gastric cancer patients. Oncotarget, 6, 8323-8338 (2015). 